\title{
CHARACTERISTICS OF LITHIUM LANTHANUM TITANATE THIN FILMS MADE BY ELECTRON BEAM EVAPORATION FROM NANOSTRUCTURED $\mathrm{La}_{0.67-\mathrm{x}} \mathrm{Li}_{3 \mathrm{x}} \mathrm{TiO}_{3}$ TARGET
}

\author{
Nguyen Nang Dinh* \\ College of Technology, Vietnam National University Hanoi, \\ 144 Xuan Thuy, Cau Giay, Hanoi, Vietnam \\ Le Dinh Trong, Pham Duy Long \\ Institute of Materials Science, Vietnamese Academy of Science and Technology, \\ 18 Hoang Quoc Viet, Cau Giay, Hanoi, Vietnam
}

Received 16 June 2007

\begin{abstract}
Bulk nanostructured perovskites of $\mathrm{La}_{0.67-\mathrm{x}} \mathrm{Li}_{3 \mathrm{x}} \mathrm{TiO}_{3}$ (LLTO) were prepared by using thermally ball-grinding from compounds of $\mathrm{La}_{2} \mathrm{O}_{3}, \mathrm{Li}_{2} \mathrm{CO}_{3}$ and $\mathrm{TiO}_{2}$. From XRD analysis, it was found that LTTO materials were crystallized with nano-size grains of an average size of $30 \mathrm{~nm}$. The bulk ionic conductivity was found strongly dependent on the $\mathrm{Li}^{+}$composition, the samples with $\mathrm{x}=0.11$ (corresponding to $\mathrm{La}_{0.56} \mathrm{Li}_{0.33} \mathrm{TiO}_{3}$ compound) have the best ionic conductivity, which is ca. $3.2 \times 10^{-3} \mathrm{~S} / \mathrm{cm}$ at room temperature. The LLTO amorphous films were made by electron beam deposition. At room temperature the smooth films have ionic conductivity of $3.5 \times 10^{-5}$ $\mathrm{S} / \mathrm{cm}$ and transmittance of $80 \%$. The optical bandgap of the films was found to be of $2.3 \mathrm{eV}$. The results have shown that the perovskite $\mathrm{La}_{0.56} \mathrm{Li}_{0.33} \mathrm{TiO}_{3}$ thin films can be used for a transparent solid electrolyte in ionic battery and in all-solid-state electrochromic devices, in particular.
\end{abstract}

Keywords: Ionic conductivity, Nanostructured LLTO, Thin film electrolyte, Electron beam deposition, Impedance spectra.

\section{INTRODUCTION}

Lithium-ion conducting materials have been received many attentions in the last years due to their potential application in solid state batteries and electrochemical devices [1-2]. Among numerous $\mathrm{Li}$ conductors, perovskite-type oxides $\left(\mathrm{ABO}_{3}\right)$ show promising lithium high ion conductivity at room temperature. Recently, many works [3-7] have shown that the new family of perovskite structure $\mathrm{La}_{0.67-\mathrm{x}} \mathrm{Li}_{3 \mathrm{x}} \mathrm{TiO}_{3}$ materials (hereafter called as LLTO) give the best lithium ionic conductors. At room temperature, the conductivity of LLTO films prepared by PLD method possesses a value up to $10^{-5} \mathrm{~S}_{\mathrm{cm}} \mathrm{cm}^{-1}[8]$, but the deposition area is conventionally small and dependent on the target size and the distance between target and substrate. Recently,

* Corresponding author email: dinhnn@ vnu.edu.vn 
$\mathrm{Li}$, Zhang and $\mathrm{Fu}[9]$ reported the preparation of LLTO thin-films by electron beam deposition, and their results showed that amorphous LLTO thin films deposited by e-beam with ionic

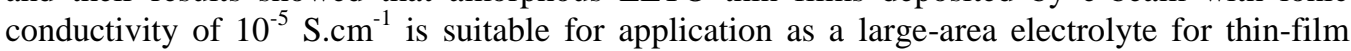
batteries. However, the LLTO film with such a low conductivity is seriously limited for application of all-solid-stated ECD which need much higher conductivity to have a high response time of display. We have reported [10] that LLTO bulk materials prepared by conventional solid-state reaction possess rather high ionic conductivity at room temperature (namely $1.5 \times 10^{-4} \mathrm{~S} / \mathrm{cm}$ ). But, the LLTO film prepared by e-beam deposition using this material as an initial target have ionic conductivity as low as $1.5 \times 10^{-7} \mathrm{~S} . \mathrm{cm}^{-1}$. In this work, the e-beam evaporation system is also used for fabricate LLTO thin films, however we used nanostructured crystalline LLTO material for the target. The powder of LLTO was prepared by thermally ballgrinding method, and then from this powder the tablet targets for electron beam deposition have been done. Structure and ionic conductivity of both the bulk and thin film samples have also been studied.

\section{EXPERIMENTAL}

The $\mathrm{La}_{0.67-\mathrm{x}} \mathrm{Li}_{3 \mathrm{x}} \mathrm{TiO}_{3}$ crystalline powder was synthesized by ball grinding method according to the following reaction:

$$
(0.67-x) \mathrm{La}_{2} \mathrm{O}_{3}+3 \mathrm{xLi}_{2} \mathrm{CO}_{3}+2 \mathrm{TiO}_{2} \rightarrow 2 \mathrm{La}_{(0.67-\mathrm{x})} \mathrm{Li}_{3 \mathrm{x}} \mathrm{TiO}_{3}+\mathrm{xCO}_{2} \uparrow
$$

where four values of $\mathrm{x}$ were chosen as $0.06,0.09,0.11$ and 0.15 . Samples corresponding to these compounds respectively were denoted by LLTO06, LLTO09, LLTO11 and LLTO15. These samples were made by following procedures: Appropriate stoichiometric amount of dehydrated $\mathrm{La}_{2} \mathrm{O}_{3}(99.9 \%), \mathrm{Li}_{2} \mathrm{CO}_{3}(99.9 \%)$ and $\mathrm{TiO}_{2}(99.95 \%)$ were purchased from Aldrich Chemicals Ltd. The mixture was dried and annealed at $800^{\circ} \mathrm{C}$ in air atmosphere for $4 \mathrm{~h}$ in order to eliminate $\mathrm{CO}_{2}$. Afterwards, the mixtures were ball-grinded by a "Fritsch Mill Model Pulverisette-6" machine, for a time duration ranging from $4 \mathrm{~h}$ to $6 \mathrm{~h}$ and $8 \mathrm{~h}$.

LLTO thin films were deposited from nanostructured LLTO targets by e-beam evaporation YBH-75-PI system with oxygen ambient. The base pressure of the evaporation chamber was 1.5 x $10^{-4} \mathrm{~Pa}$. Gas flux was regulated to maintain the chamber pressure at about $5.5 \times 10^{-2} \mathrm{~Pa}$. Corning glasses coated by metallic silver or ITO (Indium Tin Oxide) were used as substrates, the temperature of the substrates was kept at $200{ }^{\circ} \mathrm{C}$. The substrate-target distance was about 30 $\mathrm{cm}$. The deposition was maintained by use of a vibrating quartz crystal microbalance. After deposition some films were annealed in air at $450^{\circ} \mathrm{C}$ for 2 hours.

The structure and the degree of crystallization of LLTO target and thin films were characterized by X-ray diffraction (XRD) patterns recorded by a Siemens D-5000 X-ray diffractometer with $\mathrm{Cu}-\mathrm{K} \alpha$ radiation. The morphology and cross-section of the LLTO thin films were investigated by field emission scanning electron microscopy (FE-SEM). The ionic conductivity of thin films was characterized on Auto.Lab Potentiostat-PGS30 with a FRA-2 impedance spectra (IS) technique. For bulk samples, an Ag|LLTO|Ag sandwich structure was made by vacuum evaporation of Ag coatings on both surfaces of LLTO samples. For LLTO thin films, on the top of LLTO/ITO/glass the same Ag coating was also deposited. Optical measurement was carriedout on a V-570 (Jasco) photospectrometer.

\section{RESULTS AND DISCUSSION}

\subsection{Crystalline structure and ionic conductivity of powder sample}

XRD analysis shows that Li-compounds with $x$ ranging from 0.06 to 0.15 do not affect to crystalline structures of the grinded LLTO (Fig.1). 


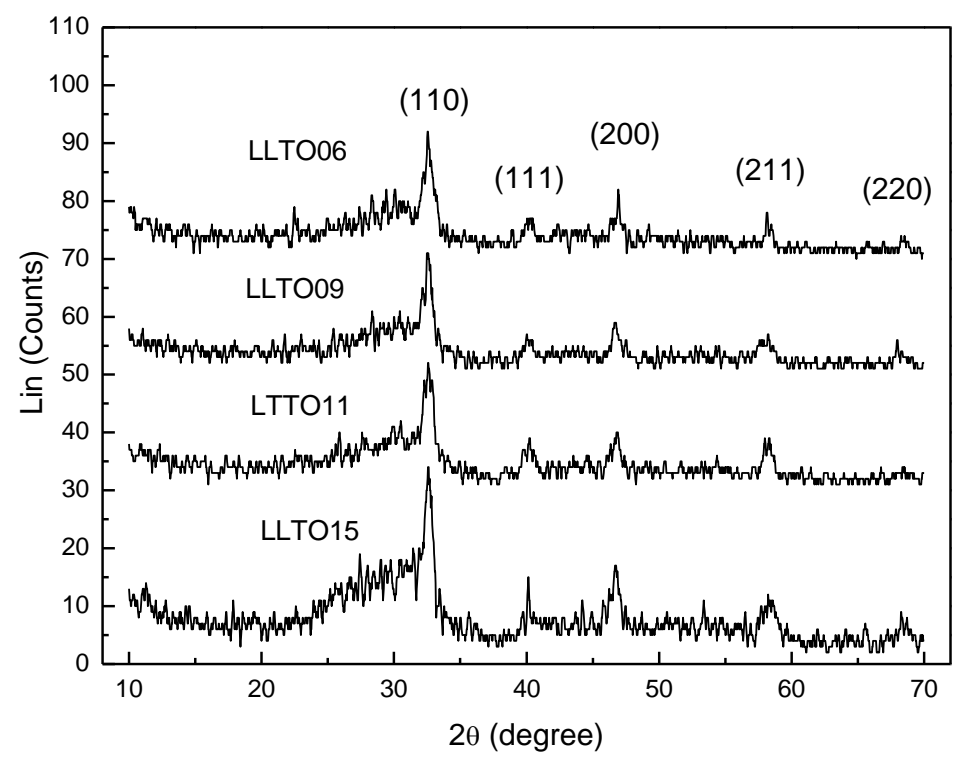

Fig. 1: XRD patterns of thermaly grinded LLTO powders with different contents of Li.

XRD patterns demonstrate that all the samples were crystallized with nanostructured powder. From the XRD peaks one can determined grain size $(\tau)$ using the Scherrer formula

$$
\tau=\frac{0.9 \lambda}{\beta \cdot \cos \theta}
$$

where $\lambda$ is wavelength of the $\mathrm{X}$-ray used, $\beta$ the peak width of half height in radians and $\theta$ the Bragg angle of the considered diffraction peak [11]. The calculation result showed that the average size of crystalline grains of (110), (111), (200) and (211) Miller indexes is rather small, namely $30 \mathrm{~nm}$. It is also shown that by the ball-grinded powder one can synthesis a uniform and well stoichiometric compound of a LLTO powder with nanostructured grains.

To measure ionic conductivity, the powder was hydrothermaly pressed to form ceramics-like pellets of $10 \mathrm{~mm}$ in diameter and $6 \mathrm{~mm}$ in thickness and then $\mathrm{Ag}$ was evaporated onto two pellet surfaces with an area (S) of $0.196 \mathrm{~cm}^{2}$. Cole-Cole plots obtained from measurements of image and real resistance parts vs. frequencies are shown in Fig. 2. The ionic conductivity of the sample is determined by formula:

$$
\sigma=\frac{\mathrm{d}}{\mathrm{R} . \mathrm{S}},
$$

where $\mathrm{d}$ is the pellet thickness, $\mathrm{S}$ is the area of the Ag-electrode deposited on the pellet surface and $\mathrm{R}$ is the solid electrolyte resistance value which is found out from equivalent scheme fitted to the Cole-Cole plots. The results of the calculation for all samples are listed in table 1.

It is known that the sample of $\mathrm{x}=0.11$ has the best ionic conductivity, which is as high as $3.2 \times 10^{-3} \mathrm{~S} / \mathrm{cm}$. The pellets made from this powder have been used for targets to electron beam deposition of LLTO thin films. 


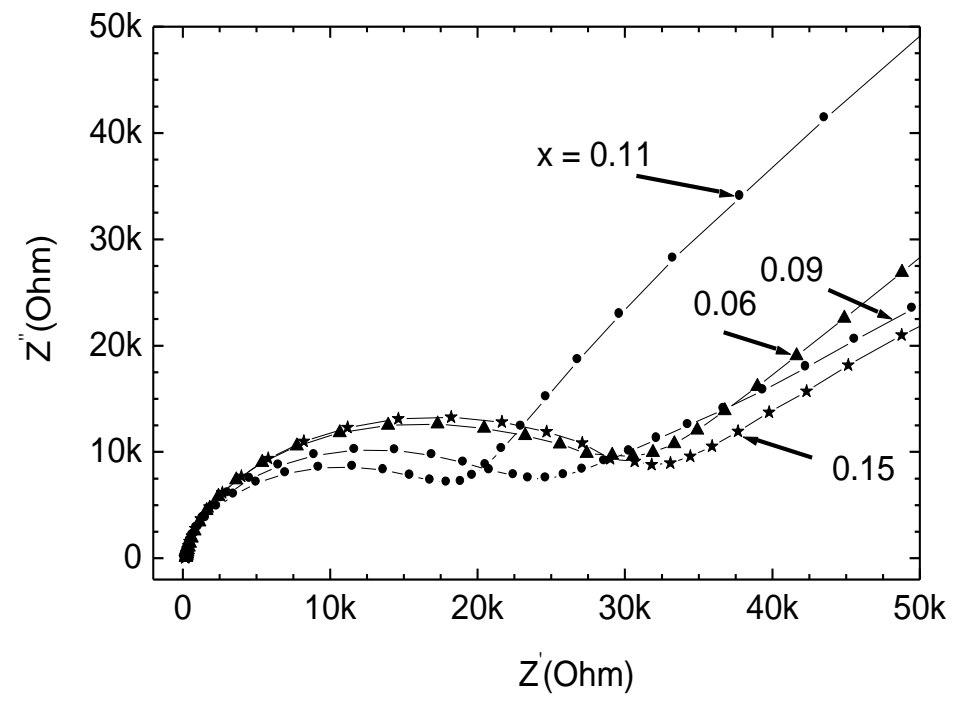

Fig. 2: Cole-Cole plots of the impedance of LLTO vs. Li contents $x=0.06(\boldsymbol{\Delta}), 0.09($ $0.11(0)$ and $0.15(*)$.

Table 1: Ionic conductivity of bulk LLTO samples vs. Li-contents.

\begin{tabular}{llll}
\hline No. & $\mathbf{x}$-values & $\mathbf{R}(\mathbf{k} \boldsymbol{\Omega})$ & $\boldsymbol{\sigma}(\mathbf{S} / \mathbf{c m})$ \\
\hline 1 & 0.06 & 3.10 & $1.8 \times 10^{-3}$ \\
2 & 0.09 & 2.26 & $2.4 \times 10^{-3}$ \\
3 & 0.11 & 1.73 & $3.2 \times 10^{-3}$ \\
4 & 0.15 & 3.46 & $1.6 \times 10^{-4}$ \\
\hline
\end{tabular}

\subsection{Morphology and ionic conductivity of LLTO thin-films}

Although targets used for electron beam deposition have crystalline structure, as-deposited LTTO11 film prepared at substrate temperature of $200^{\circ} \mathrm{C}$ exhibits noncrystalline one. Fig. 3 shows X-ray diffraction patterns of an as-deposited film (see "a" pattern). The fact that in XRD patterns there are no clear diffraction peaks, except some mound-like ones, indicate the amorphous structure of the films. This result consistent with result reported by Soon-Gil Yoon et al in [6] and Zheng-Wen Fu et al in [9], where it has been shown that the LLTO thin films deposited by pulsed laser deposition following heated treatment have not display crystalline phase until $700{ }^{\circ} \mathrm{C}$ of annealing. After being annealed at $450{ }^{\circ} \mathrm{C}$ for $2 \mathrm{~h}$, there was observed one peak corresponding to (110) crystalline grain (Fig.3, "b" pattern). However, we have seen that ionic conductivity of this film was much worse than the one of the amorphous film. Thus, further we concentrated our research on the as-deposited LLTO films.

Figure 4 shows SEM images of surface and cross-section of the LLTO11 thin film $(\mathrm{x}=0.11)$. It is seen that, the as-deposited LLTO11 films have a very dense surface with grain size less than $100 \mathrm{~nm}$ (Fig. 4a) and thickness of the film is about $270 \mathrm{~nm}$ (Fig. 4b). The film has a good adherence with the substrate. 


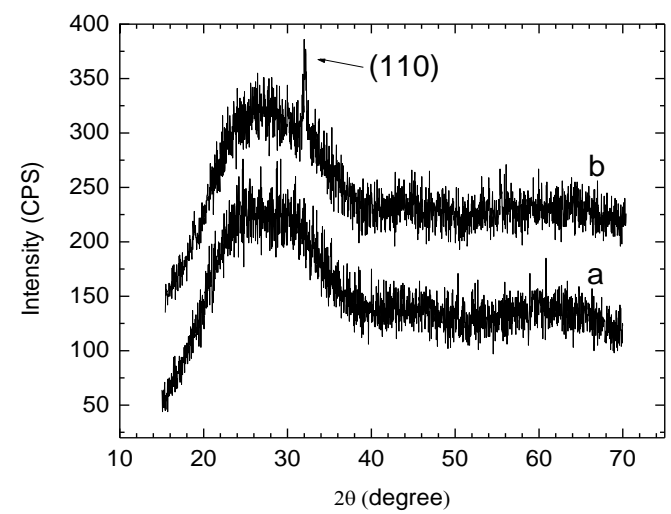

Fig. 3: XRD patterns of an as-deposited LLTO11 film (a-pattern) and after being annealed at $450{ }^{\circ} \mathrm{C}, 2 \mathrm{~h}$ (b-pattern).

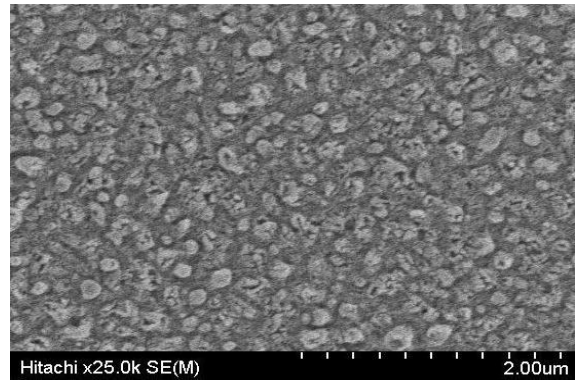

a

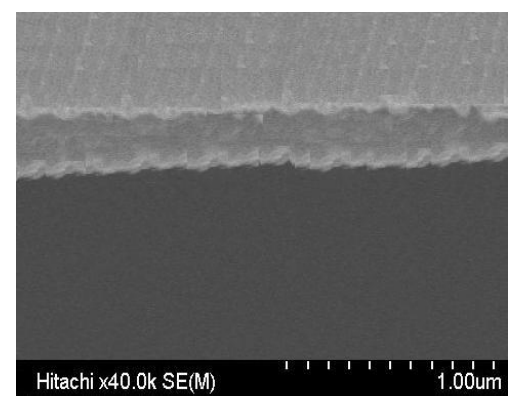

b

Fig. 4: SEM image (a) and cross-section (b) of a e-beam deposited LLTO thin film.

AC impedance spectrum of LLTO11 thin-film is shown in figure 5. This spectrum is measured at room temperature. The impedance indicates a typical behavior of single dielectric relaxation process for the ion conductor thin film sandwiched between two blocking electrodes. The impedance plot consists of two semicircles, respectively characterizing by a $\mathrm{Li}^{+}$ion conducting process in electrolyte and a process at interface of electrolyte and electrodes. The resistance of thin films was determined by using a software program for fitting experimental data with IS spectrum of equivalent circuit. As shown in recent work [12], for a thin film with thickness less than $350 \mathrm{~nm}$, to avoid error in IS elucidation it is necessary to take the influence of both the capacity of Helmholtz layer and the charge of the concentration gradient into the equivalent schemes. Thus the most suitable equivalent circuit scheme was proposed as shown in Fig. $6 \mathrm{a}$ and the IS curve fitted by this scheme is presented in Fig. $6 \mathrm{~b}$.

In part (I), $\mathrm{R}_{1}$ marks serial resistance of circuit. In part (II), $\mathrm{R}_{2}$ and $\mathrm{C}_{1}$ are the resistance of electrolyte and capacitance of the passive film of the electrolyte surface, respectively. In part (III), $\mathrm{R}_{3}$ and $\mathrm{C}_{2}$ are the resistance and capacitance of the passive film on the electrodes, respectively; $\mathrm{R}_{4}$ and $\mathrm{C}_{3}$ are resistance and double-layer capacitance of electrolyte/electrode interfaces. All values of elements obtained from the equivalent scheme are listed in table 2. 


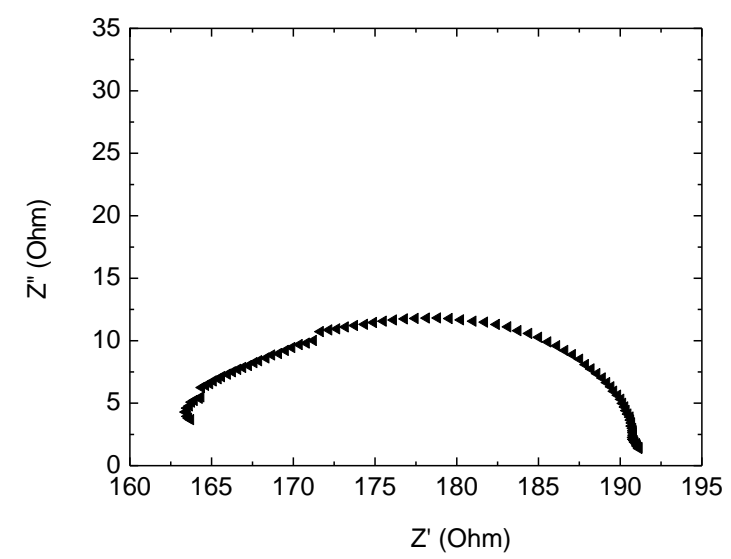

Fig. 5: The impedance plot obtained at room temperature for LLTO11 thin film.

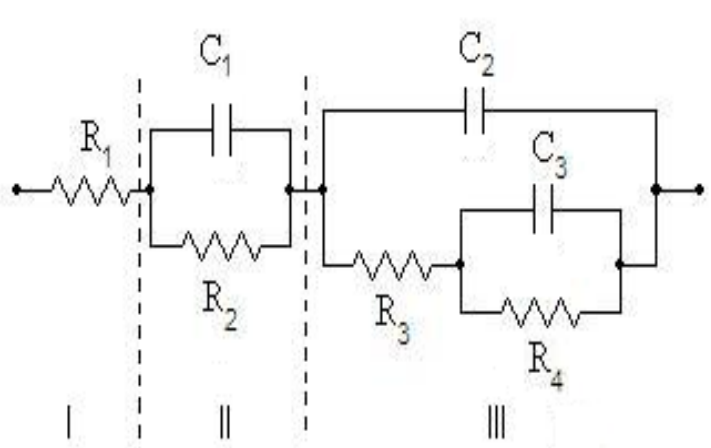

a

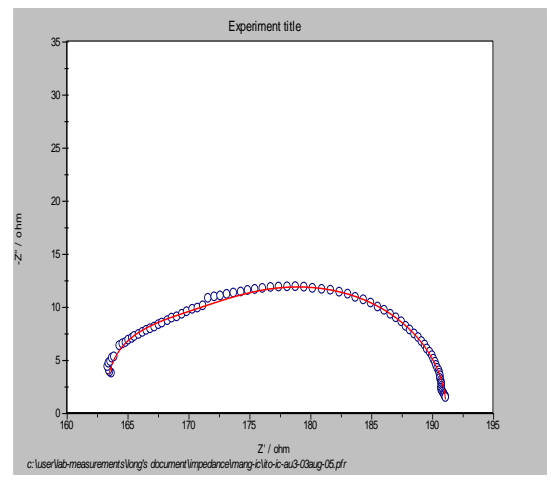

b

Fig. 6: Equivalent scheme (a) and the curve fitted to the IS spectrum (b).

Table 2: Fitted values obtained from the equivalent scheme which is shown in Fig. 6.

\begin{tabular}{|l|l|l|l|l|l|l|l|}
\hline Element & $\mathrm{R} 1(\Omega)$ & $\mathrm{R} 2(\Omega)$ & $\mathrm{C} 1(\mathrm{nF})$ & $\mathrm{C} 2(\mathrm{nF})$ & $\mathrm{R} 3(\Omega)$ & $\mathrm{R} 4(\Omega)$ & $\mathrm{C} 3(\mathrm{nF})$ \\
\hline Value & 163.3 & 8.7 & 163.0 & 225.7 & 6.54 & 25.45 & 157.7 \\
\hline
\end{tabular}

The ionic conductivity of the film $\left(\sigma_{\mathrm{Li}}\right)$ can be calculated from the value of electrolyte resistance $\mathrm{R}_{2}$ according to the formula (3). In this case $d$ is the thickness of the deposited film $(\mathrm{d}=270$ $\mathrm{nm}), \mathrm{S}$ is the Ag-electrode area $\left(\mathrm{S}=0.09 \mathrm{~cm}^{2}\right)$. Thus at room temperature, ionic conductivity of the films measured has a value as high as $\sigma_{\mathrm{Li}}=3.5 \times 10^{-5} \mathrm{~S} / \mathrm{cm}$, that is comparative with the conductivity of the LLTO thin films prepared by the pulsed laser deposition [8]. In comparison with bulk conductivity of corresponding target, the ionic conductivity of thin films is lower in two orders by magnitude. Such ion conducting decrease of amorphous thin film could be originated from (i) decomposition of a part of lithium amount during evaporation process caused a lack of Li in LLTO thin films, (ii) the absence of gain boundaries, and (iii) increasing interface effect. 


\subsection{Optical property}

As-deposited LLTO11 thin films have slightly yellow colour in visible range. Figure 7 shows transmittance spectrum of the film in wavelength range from $350 \mathrm{~nm}$ to $850 \mathrm{~nm}$. Transmittance spectra of thin films is rather high in visible range, it is of 80 to $90 \%$. From the optical transmittance spectra one can evaluate optical bandgap $\left(E_{g}\right)$ of the material. In case reflectance is negligible, absorption coefficient $(\alpha)$ can be determined by well-known formula:

$$
\alpha=\frac{1}{d} \times \ln \left(\frac{1}{T}\right) .
$$

Using formula of relationship between $\alpha$ and photon energy (hv), as bellow [13]:

$$
\alpha h v=B\left(h v-E_{g}\right)^{2}
$$

where $\mathrm{B}$ is a constant coefficient, one can plot a curve of $(\alpha h v)^{1 / 2}$ vs. hv. From this dependence, $\mathrm{E}_{\mathrm{g}}$ can be determined as a value of the photon energy which corresponds to the case $\alpha h v \rightarrow 0$. Figure 8 shows the plot obtained from equation (5), consequently $\mathrm{E}_{\mathrm{g}}$ of the LLTO11 film is found to be of $2.3 \mathrm{eV}$. This shows that the films prepared by electron beam deposition from nanostructured LLTO11 target exhibit both the high Li-ionic conductivity and the high transmittance.

Fig. 7: Transmittance spectra of an amorphous LLTO $(x=0.11)$ thin film.

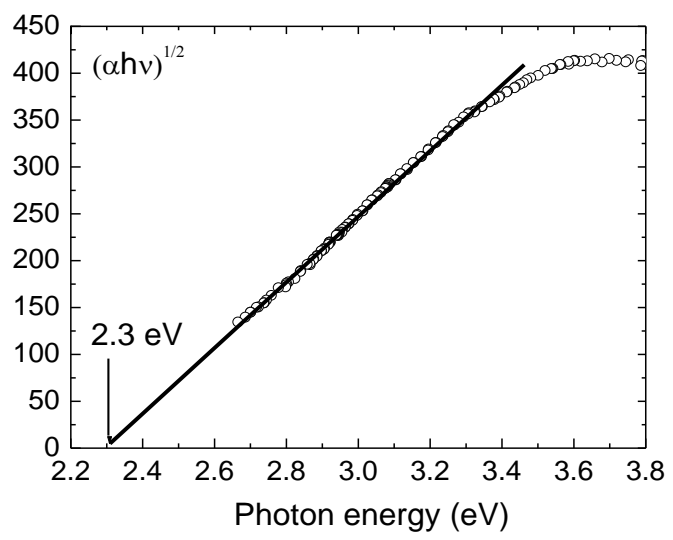

Fig. 8: Absorption coefficient vs. photon energy plot for determination of $E_{g}$ of the LLTO film. 


\section{CONCLUSION}

By using thermally ball-grinding method, nanostructured LLTO powder was synthesized. The best ionic conductivity of the bulk $\mathrm{La}_{0.67-\mathrm{x}} \mathrm{Li}_{3 \mathrm{x}} \mathrm{TiO}_{3}$ ceramic-like samples was obtained for $\mathrm{x}=$ 0.11 , it is as high as $3.2 \times 10^{-3} \mathrm{~S} / \mathrm{cm}$. LLTO solid state electrolyte thin films were made by electron beam deposition from nano-crystalline targets. At room temperature, the ion conductivity of the LLTO11 films was found to be of a value of $3.5 \times 10^{-5} \mathrm{~S} / \mathrm{cm}$. From the fact that the films exhibit both high transmittance and high conductivity one can suggest application of the LLTO thin films in solid electrolyte for ion batteries and for all-solid-state electrochromic devices, in particular.

\section{ACKNOWLEDGEMENT}

The authors express sincere thanks to MSc. Tran Dang Thanh and MSc Do Hung Manh, Institute of Materials Science, Vietnam Academy of Science and Technology, respectively for $\mathrm{XRD}$ analysis and FE-SEM characterization. This work was supported by the Vietnam National Program for researches in Natural Science (Project Code: 410306).

\section{REFERENCES}

1. Belous, A.G., Novitskaya, G.N., Polyanetskaya, S.V., and Yu. I. Gormikov (1987), Izv. Akad. Nauk, USSR, Neorg. Mater. vol. 23, pp. 470-479.

2. Inaguma, Y., Chen, L., Itoh, M., Nakamura, T., Uchida, T., Ikuta, M., and Wakihara, M. (1993), Solid State Commun., vol. 86, pp. 689-671.

3. Belous, A.G. (2001), Journal of the European Ceramic Society, vol. 21, pp. 1797-1800.

4. Bonhke, O., Emerym J., and Fouquetm J.L. (2003), Solid State Ionics, vol.158, pp. 119-132.

5. Li, J., Wen, Z., Xu, X., and Zhu, X. (2005), Solid State Ionics, vol. 176, pp. 2269-2273.

6. Ahn, J.K. and Yoon, S.G. (2004), Electrochimica Acta, vol. 50 , pp. 371-374.

7. Furusawa, S., Tabuchi, H., Sugiyama, T., Tao, S., and Irvine, J.T.S. (2005), Solid State Ionic, vol. 176 , pp. 553-558.

8. Ahn, J.K. and Yoon, S.G. (2005), Electrochem. Solid-State Lett, vol. 8(2), A75-A79.

9. Li, C.L., Zhang, B., and Fu, Z.W. (2006), Thin Solid Films, vol. 515, pp 1886-1898.

10. Dinh, N.N., Long, P.D., and Trong, L.D. (2004), Commun. in Phys., Vol. 14/2, pp. 90-96.

11. Cullity, B.D. (1978), Elements of X-Ray diffraction, 2nd ed., p.102, Addison-Wesley Publishing Company, Inc., Reading, MA.

12. Dinh, N.N., Ngoc, N.T.B., and Trong, L.D. (2006), VNUH J. Sci., Mathematics-Physics, Vol. 22/1, pp. 54-61.

13. Willardson, R.K. and Beer, A.C. (1967), Semiconductors and Semimetals, Vol. 3 Optical properties of III-V compounds (Chapter 6), Acad. Press, New York - London. 